\title{
Philosophiques
}

\section{Johannès B. Lotz, Martin Heidegger et Thomas d'Aquin traduit par de l'allemand Philibert Secretan, Paris, PUF, 1988, 232 p. (coll. Théologiques).}

\section{Bertrand Rioux}

Volume 16, numéro 2, automne 1989

URI : https://id.erudit.org/iderudit/027091ar

DOI : https://doi.org/10.7202/027091ar

Aller au sommaire du numéro

Éditeur(s)

Société de philosophie du Québec

\section{ISSN}

0316-2923 (imprimé)

1492-1391 (numérique)

Découvrir la revue

Citer ce compte rendu

Rioux, B. (1989). Compte rendu de [Johannès B. Lotz, Martin Heidegger et Thomas d'Aquin traduit par de l'allemand Philibert Secretan, Paris, PUF, 1988, 232 p. (coll. Théologiques).] Philosophiques, 16(2), 418-422.

https://doi.org/10.7202/027091ar d'utilisation que vous pouvez consulter en ligne.

https://apropos.erudit.org/fr/usagers/politique-dutilisation/ 
JOHANNÈS B. LOTZ, Martin Heidegger et Thomas d'Aquin traduit de l'allemand par Philibert Secretan, Paris, PUF, 1988, 232 p. (coll. Théologiques).

$$
\text { par Bertrand Rioux }
$$

Le public français connaît déjà quelques ouvrages du P. Lotz dont l'excellent livre Le jugement et l'Être où l'on trouve une discussion remarquable avec Heidegger auteur de la conception du jugement (pp. 203-224). Le présent ouvrage qui vient d'être traduit de l'allemand, date de 1975, ce qui explique sans doute que l'on ne trouve aucune prise de position sur l'engagement politique de Heidegger en rapport avec sa pensée philosophique. Pourtant, même si l'on veut mener un débat serein sur cette pensée dont l'influence a été énorme et qui n'est comparable à nulle autre au $\mathrm{XX}^{\mathrm{e}}$ siècle, il n'est plus possible désormais d'ignorer cette question. Le débat philosophique est mené a vec vigueur et rigueur entre deux des plus grands représentants d'une philosophie de l'être. Pour ceux qui connaissent la pensée de Thomas d'Aquin et de Heidegger et qui voudraient saisir l'occasion pour mieux 
connaître l'une er l'autre, ils trouveront un grand profit à éclairer l'un par l'autre ces deux grands penseurs. Si paradoxal que cela puisse paraître, il nous semble que c'est la pensée de Thomas d'Aquin qui peut le mieux rendre justice à l'interrogation de l'être par Heidegger même si elle en diffère profondément.

Le livre de Lotz comprend quatre essais. Le quatrième traité « qui donne à cet ensemble son vrai relief, est précédé de trois essais plus brefs, le tout progressant du plus simple au plus difficile, du plus sommaire au plus détaillé » (p. X). Le premier essai porte sur l'essence de l'homme selon Heidegger. Répondant aux questions de Jean Beaufret sur l'humanisme et les fondements d'une éthique, Heidegger montre que l'homme se définit par l'ek-sistence en tant qu'il " est entièrement référé à l'être et déterminé par l'être », qu'il n'est pas « un pour-soi mais un pour-l'être» (p. 3). Si l'essence de la technique est "le suprême danger ", c'est parce qu'en elle le rapport de l'homme à l'être est complètement obscurci et recouvert. Elle achève ainsi l'oubli de l'être qui caractérise la métaphysique et suscite le nihilisme et l'athéisme. La crise de Dieu est une crise de l'être. L'homme ne peut être sauvé que s'il effectue «un retour au fondement », c'est-à-dire à l'être.

C'est sur ce terrain commun de la question de l'être comme fondement de l'étant qu'a lieu la rencontre des deux grands penseurs dont parlent le deuxième et le troisième traité. Contrairement à l'opinion accréditée par Heidegger lui-même, l'Aquinate "pense thématiquement l'être" (p. 69), même si «aucun philosophe n'a autant thématisé la question de l'être que Heidegger » et alors que "c'est bien sous son impulsion que nous avons renouvelé notre regard sur l'être tel qu'en a traité Thomas d'Aquin » (p. 27). Peut-on dire que Heidegger a posé la question de l'être d'une façon plus fondamentale que Thomas d'Aquin alors que ce dernier a eu une vision plus vaste de l'être en découvrant le soi le plus interne de l'être dans l'Être subsistant de Dieu? Il est difficile de dire les choses ainsi lorsque toutes les grandes thèses de Thomas d'Aquin sur la nature de l'être, sur la composition de l'étant fini selon les deux principes de l'essence et de l'esse, sur le nom propre de Dieu comme Celui qui est et sur la création sur le plan philosophique comme le don de l'être (esse) sont la thématisation explicite de l'être comme l'acte d'exister-actus essendi-exercé par l'étant. Si Thomas d'Aquin parvient au Soi le plus ultime de l'être, c'est que l'être est thématisé au-delà de la manifesteté de l'étant et de la phénoménalisation de l'être.

Dans le cadre de l'analyse existentiale, l'essence du fondement consiste dans la liberté finie du Dasein qui projette l'être comme monde (49) alors que dans la différence ontologique l'être se montre comme " »le fondement de la manifesté de l'étant ». La métaphysique a pensé l'éclairement de l'étant sans le rapporter à l'être. Par ailleurs, dans la Lettre sur l'bumanisme, Heidegger montre bien que le projet de l'homme est un projet jeté et que l'être est le fondement de l'homme (52). Ce dernier n'est pour lui-même qu'en étant pour l'être. L'éclairement de l'être en tant que copropriation - Ereignis de l'homme et de l'être, fonde l'apparition de tout étant, y compris du divin et de Dieu. 
Qu'en est-il pour Thomas d'Aquin? L'étant est par l'être qui le fonde. Si l'essence est le fondement proche, l'être lui est le fondement ultime. Et l'être nous est connu d'abord comme l'acte de l'étant sensible. Il faut noter que la notion d'esse a plusieurs sens : 1) celui d'essence (ou quidditas cu natura) qui enveloppe les diverses catégories de l'être 2) celui d'actus essentiae et 3) celui de la vérité de la proposition. C'est le deuxième sens qui prête à a mbiguïté. En effet, il faut le comprendre soit comme l'actualité de fait de l'essence, c'est le sens aristotélicien, soit encore et d'une manière proprement métaphysique comme l'acte de toute forme, intérieur à l'essence mais qui la transcende. L'essence n'est ainsi qu'une participation de cet acte d'être qui se réalise diversement et proportionnellement à l'essence qui le reçoit et le limite. Il s'agit d'une actualité ontologique élevée à la compréhension d'un principe et d'un fondement de l'existence empirique des choses. Nous sommes loin de l'être conçu dans le mode ontique de la réalisation de l'essence et comme passage de l'état de possibiblité à l'état de réalité par l'effet d'une causalité efficiente, par exemple Dieu. Heidegger pense dans le mode phénoménologique de l'apparition la transcendance de l'être immanent à l'étant, alors que Thomas d'Aquin ne réduit pas l'être au fondement de la manifesteté de l'étant selon l'épokè préliminaire de la vérité de l'être comme dévoilement, mais le saisit en le posant et en l'affirmant dans le jugement comme un fondement de réalité de l'étant dans l'étant. L'être est manifesté à l'homme et pour l'homme comme un fondement qui fait réel l'étant en tant qu'il exerce pour lui-même et indépendamment de l'homme qui le rend manifeste un acte qui est immanent à l'étant. Cette fondation dans l'être est contenue dans les choses sur un mode non réflexif et inconscient (62). La fondation réflexive et consciente telle que l'homme l'effectue est entièrement rapportée à la fondation de réalité de l'étant. L'origine de cette fondation n'est pas finalement dans l'homme, mais dans l'être. L'être qui est fondement immanent - transcendant de l'étant indique et exige un fondement purement transcendant de l'étant qui est l'être subsistant, l'être qui repose et subsiste en soi. Ce n'est que si Dieu existe comme plénitude absolue que la différence de l'être et de l'étant peut être éprouvée par l'homme, que l'être est le fondement de I'homme et qu'il y a un monde d'étants où l'être est mêlé à l'étant. La finitude de l'être demande à être pensée comme contingence ontologique de cequi-est-avec-néant sur le fondement causal de ce qui-est-sans-néant pouvonsnous dire. La causalité et la différence sont pensées par Thomas dans le registre ontologique d'une dépendance quant à une fondation de l'étant dans l'être et non seulement d'une dépendance des étants entre eux. Dieu luimême n'est appelé un primum ens qu'en tant qu'il est ipsum esse subsistens. De plus, si Dieu est cause des étants en donnant l'être (esse), parce qu'il est souveraine liberté, il n'a pas de soi la raison de cause et donc de première cause. Son nom propre est l'aséité, Celui qui est. Il n'est ni causa sui, ni fondé en quelque chose d'extérieur à son être. Mais si l'être ne se cause pas luimême et n'est causé par rien d'autre, il reste qu'il doit se fonder lui-même (64), qu'il doit être son propre fondement, sans quoi il ne serait qu'un pur fait sans nécessité. Il ne se donnerait à comprendre que comme jeu et se supprimerait lui-même (63). Quand Thomas écrit que «Deus est esse 
tantum », il ne dit pas que Dieu n'as pas d'essence, mais qu' « en Dieu l'essence et l'existence soient identiques" de sorte que l'essence de Dieu est d'exister. Par soi, Dieu est son propre fondement et fonde par ailleurs tous les étants qui ne sont que par participation. C'est ainsi qu'il est mystérieux et c'est comme Personne infinie et absolue qu'il est l'Être pur et non comme un sansfond absurde qui se détruirait lui-même. C'est pourquoi, l'interpellation de I'homme par l'être est ultimement une interpellation personnelle demandant une réponse libre. «Là, écrit Lotz, la philosophie rencontre celui que la religion honore et adore comme Dieu.» (p. 70)

Le dernier traité porte sur le thème central du temps dans l'œuvre de Heidegger. Lotz conclut que «malgré tout ce qui rapproche les deux penseurs, un motif plus fondamental les sépare: Heidegger ne dépasse pas l'horizon du temps, alors que Thomas d'Aquin voit le temps comme participation à l'éternité » (p. X). Le texte de Lotz est particulièrement riche et développé. Je n'en soulignerai que quelques aspects importants. L'anthropologie philosophique de Thomas d'Aquin définit l'homme aux confins du temps et de l'éternité en tant qu'il est un esprit incarné, au plus bas degré de l'esprit et au plus haut degré de la matière. Les opérations de l'esprit en nous sont effectivement mais indirectement ou extrinsèquement soumises au temps en tant qu'elles puisent leur objet directement dans le monde matériel et qu'elles sont soumises aussi aux opérations de la vie sensible, des sens et de l'imagination. Mais en elles-mêmes, les opérations spirituelles sont au-delà du temps. De même qu'il n'y a des étants que par l'être qui transcende l'étant et qui de soi peut se réaliser selon la plénitude absolue de l'acte d'être, ainsi il n'y a de temps que dans le voisinage de la pensée qui comme telle émerge audessus du temps. Alors que le temps se suffit à lui-même chez Heidegger, pour l'Aquinate il requiert un autre principe qui l'unifie et le fonde de sorte qu'il ne saurait être l'ultime horizon de la compréhension de l'être. Alors que le temps se constitue lui-même chez Heidegger, il est constitué chez Thomas par un principe qui le transcende et qui lui-même doit être intemporel (p. 109-110). Si le temps est fondé pour Aristote et l'Aquinate dans le mouvement ou dans le devenir, il se constitue dans l'activité de l'âme numérante, de sorte qu'il n'y a de temps à proprement parler que par l'homme et pour l'homme. Il n'y a d'expérience du temps proprement dit que là où l'esprit élève «le successif instinctivement vécu ", la mémoire toute sensible à la réminiscence consciente et réfléchie. Grâce à la distinction profonde dans la connaissance intellectuelle humaine de la raison et de l'intellect comme deux aspects de la même faculté de connaissance, il y a une raison tranporelle qui est engagée dans le monde sensible et un intellect supra-temporel qui «inaugure l'éternité qui à son tour et d'abord rend possible le temps" (p. 123). L'horizon de l'être est l'éternité parce que la saisie de l'être comme actus essendi dépasse le rapport de fondation à l'étant temporel. Être n'est pas identique au mode temporel d'être, car dans sa racine la plus intime d'acte ultime au fondement de l'étant il aspire à se réaliser audelà de tout devenir, dans le maintenant de l'éternité. De même qu'il n'y aurair pas la transcendance de l'être qui fonde l'homme comme voué à 
l'apparition de l'être et l'être mêlé à tout étant sans la transcendance séparée de l'Être subsistant, ainsi il n'y aurait pas d'étants temporels ni de temporalité du Dasein et de l'être lui-même, ni d'histoire de l'être, si la durée propre de l'être et de l'esprit n'était pas l'éternité. Il faut alors distinguer trois durées : la durée successive et continue du mouvement dans l'être, c'est la durée temporelle; la durée discontinue de l'esprit comme esprit qui comporte des changements dans l'objet des opérations et qui est «la persévérance dans l'être des actes spirituels d'intellection ou de saisie contemplative, qui est ainsi «composée d'instants supérieurs au temps» ou d'une «succession de fragments d'éternité » selon l'expression de J. Maintain; enfin, la durée proprement éternelle par distinction de l'aevum, de l'éviternité décrite plus haut, d'un maintenant d'une plénitude parfaite qui ne comprend ni changement et ni devenir d'aucune sorte.

Enfin, le Père Lotz institue un débat entre l'Ereignis de Heidegger et la création de Thomas d'Aquin. Alors que Heidegger en reste au moment phénoménologique de l'Ereignis d'où tout étant provient, y compris le divin et Dieu, Thomas d'Aquin fait intervenir au plan philosophique lui-même "l'événement " de la création pour expliquer que des êtres-qui-sont-avec-néant n'ont l'être réel et non pas seulement l'être phénoménalisé dans le procès d'apparition pour nous que parce que Dieu comme Celui qui est leur donne d'être participativement à son Être. La création n'est temporelle que pour le créé et en tant que voulu par Dieu éternellement. Il y a bien ainsi une origine à l'Ereignis dans l'Être séparé de Dieu.

Département de pbilosopbie

Université de Montréal 